\title{
Physicochemical Properties of $\mathrm{CuO}_{2}(\text { acac })_{2}$ Supported on Functionalized MCM41 Containing Thiourea Ligand
}

\author{
Amirah Ahmad, Hamizah Md. Rasid, and Karimah Kassim
}

\begin{abstract}
A new material is prepared by encapsulating functionalized-MCM41 with 2-hydroxy-2-mercaptopyrimidine (2-thiouracil) and copper acetate monohydrate. The functionalized-MCM41 was first synthesized by using Ludox as silica source and cetyltrimethylammonium bromide (CTABr) as template and was modified via co-condensation method using 3-aminopropyltriethoxysilane (APTES). The surface and textural of synthesized material have been characterized by using X-ray diffraction (XRD), $\mathbf{N}_{2}$ adsorption isotherms, BET surface area measurement, FT-IR, elemental analyzer and scanning electron microscopy (SEM). The low-angle XRD results indicated that the $\mathrm{CuO}_{2}$ (acac)-Thio-APS-MCM41has similar XRD pattern as the mesoporous MCM41 although the intensity decreases. For high-angle XRD, the results show the presence of monoclinic $\mathrm{CuO}$ at $2 \theta=35.3$ and $38.6^{\circ}$ while $\mathrm{N}_{2}$ adsorption and BET results were revealed that the $\mathrm{CuO}_{2}$ (acac)-Thio-APS-MCM41 possessed a good mesoporous structural ordering and uniform mesoporosity. FTIR spectra confirmed that the organosilane group still retained in the pore channel of the $\mathrm{CuO}_{2}$ (acac)-Thio-APS-MCM41 even after modification and the band of $\mathrm{C}=\mathrm{S}$ and $\mathrm{Cu}=\mathrm{O}$ also appeared in $\mathrm{CuO}_{2}$ (acac)-Thio-APS-MCM41 spectra with the presence of thiourea ligand and copper. Hence, the characterization results were revealed that the synthesized material has an ordered hexagonal structure, a narrow pore size distribution, a high surface area and thiourea complex were successfully grafted onto the pores of functionalized-MCM41.
\end{abstract}

Index Terms-MCM41, Monoclinic, Thiouracil, Thiourea.

\section{INTRODUCTION}

Mesoporous M41S family consists of several unique members having different mesophases. The three important members are MCM41 which has a hexagonal arrangement of unidimensional pore channels, MCM48 which has a three dimensional cubic pore structure and MCM50 which has unstable lamellar structure. Among the M41S materials, MCM41 has received the most attention because of its simple structure, ease of synthesis, high surface area, large pore volume, ordered pore arrangement and very narrow pore diameter. Increasing attention has been paid to M41S family since the researchers at Mobil Research and Development Corporation first reported the synthesis of MCM41 [1]. Therefore, MCM41 are found to be important in many fields such as catalysts, separation and adsorption [2].

However, pure MCM41 showed very limited catalytic activity due to the lack of lattice defect, acidity or basicity and redox properties. Hence, it requires modification to introduce other features into mesoporous silicates to improve their properties. Incorporation of heteroatoms into the framework or onto the wall or onto the pore of mesoporous silicate can increase the amounts of defects, the acidity or basicity, the redox property and thus improve the catalytic ability of silicate [3]. By modification of surface MCM-41 with organic groups, it can improve the catalytic activity by raising their hydrophobicity [4] using 3-aminopropyltriethoxysilane (APTES). Zhao and Lu* (1998) was studied the modification of MCM41 by surface silylation with trimethylchlorosilane and adsorption study. They found the surface chemistry of MCM41 can be effectively modified to be more hydrophobic by chemical attachment of organic species and be an effective technique in selective adsorbents for the removal of organic compounds from streams or wastewater [5].

Besides, there are only a few catalytic active sites on MCM41 wall. Therefore, in order to utilize this material for catalysis, it is necessary to generate the appropriate catalytically active sites to the mesoporous silicates regarding its application. Many transition metals have been incorporated into the wall structure such as $\mathrm{Ti}, \mathrm{Cr}, \mathrm{Al}$, and $\mathrm{V}$ to endow the mesoporous materials with catalytic properties especially in the selective oxidation, epoxidation, alkylation and isomerisation of organic compounds [6]. Furthermore, the modification of silica surface and related materials with specific ligand fields or metal complexes creates a powerful new capability for catalysis, chemical separations and sensor development. Tangestaninejad et. Al., (2009) was reported the molybdenum supported on functionalized MCM41 containing Schiff Base ligand as an efficient and reusable catalyst for alkenes epoxidation with tert-BuOOH [7].

Here, we describe the immobilization of 2-thiouracil on functionalized-MCM41 to form thiourea ligand and as potential donor ligand for transition metal ion to form supported metal thiourea complex (Fig. 1). The 2-thiouracil, (2-hydroxy-2-mercaptopyrimidine) is a pyrimidine derivative of well known biological properties and can exist in six tautomeric forms. Structures of the samples were characterized by XRD, FT-IR, Elemental analyzer, $\mathrm{N}_{2}$ adsorption, BET surface area and FESEM techniques.

\section{PROCEDURE}

work was supported by the Ministry of Science, Technology and Innovation Malaysia (MOSTI) under Grant FRGS-600-RMI/ST/FRGS5/3/Fst $(41 / 2010)$

The authors are with Faculty of Applied Sciences, Universiti Teknologi MARA, Malaysia (my_silberberg@yahoo.com.my)

\section{urely Siliceous MCM41}

The synthesis of MCM-41 host matrix is carried out 
according to the molar composition below:

$$
6 \mathrm{SiO}_{2}: \mathrm{CTABr}: 1.5 \mathrm{NaOH}: 0.15\left(\mathrm{NH}_{4}\right)_{2} \mathrm{O}: 250 \mathrm{H}_{2} \mathrm{O}
$$

The sodium silicate solution was prepared by mixing Ludox with sodium hydroxide in double distillated water. Then, the resulting solution is stirring for 2 hours. In another polypropylene bottle, cetyltrimethylammonium bromide was dissolved in double distillated water and ammonium hydroxide aqueous solution was added. The mixture was heated and stirred for 1 hour. After that, the sodium silicate solution (PART A) was added into the propylene bottle containing the template solution (PART B) and then simultaneously stirred vigorously. The resulting mixture was age overnight. Then the precipitate was filtered, washed with doubly distillated water and dried in oven. The final step involves the calcinations of the as-synthesized sample at $550{ }^{\circ} \mathrm{C}$ to remove the organic template.

\section{B. Synthesis of Aminopropylated MCM41}

Aminopropylated MCM41 was prepared by mixing MCM41 (2.0 g), 3-aminopropyltriethoxysilane $(2.2 \mathrm{~mL})$ in $\mathrm{n}$-Hexane $(50 \mathrm{~mL})$. Then, the mixture was refluxed for 6 hours The mixture was filtered, washed, dried at room temperature and stored in desiccators [8].

\section{Synthesis of Thiourea Ligand Grafted APS-MCM4I}

The thiourea complex grafted APS-MCM41 was prepared as following: APS-MCM41 was added to a solution of thiouracil $(2.5 \mathrm{~g})$ in $\mathrm{n}$-Hexane $(50 \mathrm{~mL})$ and refluxed for 24 hours. The mixture was filtered, washed and dried under vacuum overnight.

\section{Synthesis of CuO2(acac)-Thio-APS-MCM41}

To a solution of $\mathrm{CuO}_{2}(\mathrm{acac})_{2}(2.5 \mathrm{~g})$ in n-Hexane $(50 \mathrm{~mL})$ was added Thio-APS-MCM41 (2.5 g) and refluxed for 24 hours. The mixture was separated by filtration, washed and dried under vacuum.

\section{E. Characterization}

Several characterization techniques have been utilized in order to elucidate and provide structural information and physicochemical properties of the synthesized material. X-Ray diffraction (XRD) patterns of the samples were obtained by using Rigaku D/max-2500 powder diffractometer with $\mathrm{Cu}-\mathrm{K} \alpha$ source $(\lambda=1.5418 \AA, 40 \mathrm{kV}, 40 \mathrm{~mA})$. The sampling for this analysis is made by grinding sample manually into fine powder to fit into sample holder with an area of 10-15 diameters and approximate depth of $1 \mathrm{~mm}$ in a glass plate $(40 \times 33 \times 2 \mathrm{~mm})$ The depression is completely filled with the sample and the surface of the powder was flattened to the level of the glass plate using a glass microscope slide to ensure the correct sample weight within the diffractometer. The particle size and morphology of the samples were characterized using a Zeiss Supra 35VP Field emission scanning electron microscopy (SEM). Samples in a powder form were mounted over carbon stubs using double sided tape. The carbon analysis of the samples was acquired by using Flash 1100 Organic Elemental Analyzer Thermo Finnigan. The FT-IR spectra were obtained on a Perkin-Elmer Spectrum One FTIR spectrometer. The finely ground sample is well-mixed with $\mathrm{KBr}$ powder and the mixture is then placed between two $13 \mathrm{~mm}$ evacuable die under 10 tons of pressure for 2 minutes to form a transparent pellet. $\mathrm{N}_{2}$ adsorption isotherm and BET surface area were measured at 77K on a Micromeritics ASAP 2010 Volumetric Adsorption Analyzer. The samplea was outgassed at temperature $200{ }^{\circ} \mathrm{C}$ for an hour for dehydration purpose. The adsorption and desorption process carried out for a few times until constant value were obtained.
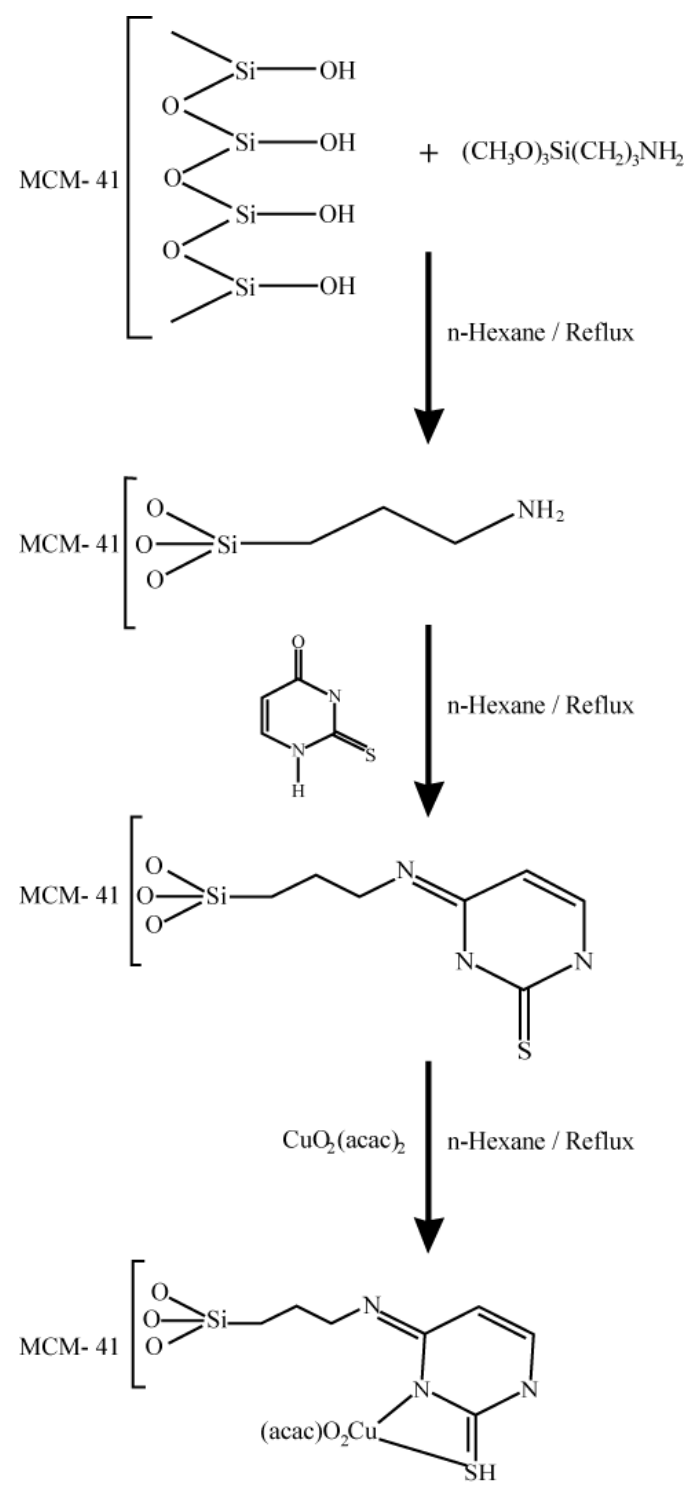

Fig. 1. Preparation of $\mathrm{CuO}_{2}$ (acac)-Thio-APS-MCM41

\section{RESULTS AND DISCUSSION}

\section{A. XRD Patterns}

The XRD patterns of the synthesized material are shown in Fig. 2 and 3. The low angle XRD patterns show three resolved peaks that can be indexed to the (100), (110) and (200) reflections indicating the characteristics of hexagonal ordered MCM41 structure [9]. These peaks were obviously observed in the XRD patterns of the synthesized materials (Fig. 2). High intensity of the main peak (100) shows high degree of long-range order and existence of uniform pores in the synthesized material. The XRD peak intensities of the synthesized materials decreased when the pores were filled 
with organic functionalized, thiourea ligand and metal (Fig. 2 (b), (c), (d)). This might be due to the decreased of the long range order of the mesoporous sample whereby the addition of amino, thiourea and metal into the gel mixture will lead to some structural rearrangement. However, XRD results show that the mesopores structures still remained intact as hexagonal structure. Moreover, high angle XRD patterns shows the peak in between $2 \theta=20^{\circ}-100^{\circ}$ after addition od thiouracil and metal (Fig. 3). The peaks at $2 \theta=35.28^{\circ}$ and $38.6^{\circ}$, corresponding to monoclinic $\mathrm{CuO}$ (Fig. 3 (b)) [10].

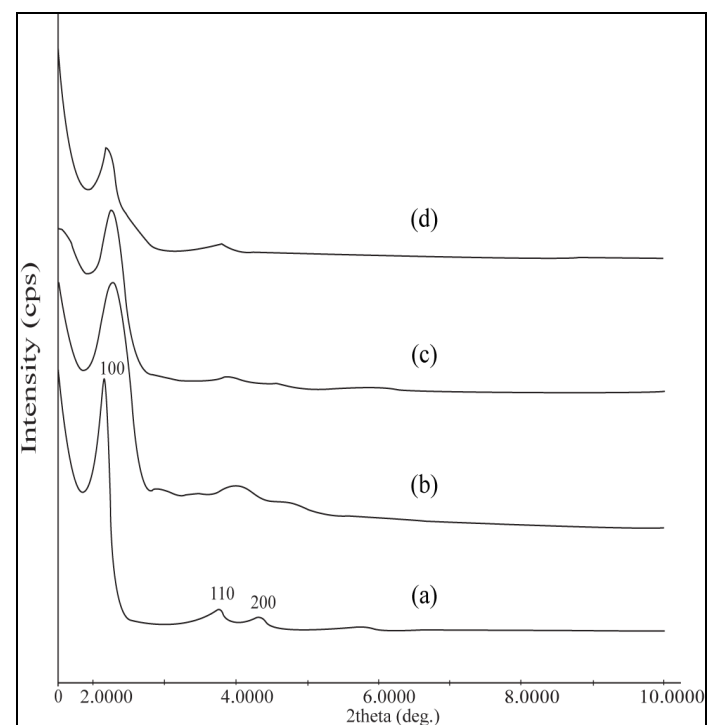

Fig. 2. Low angle XRD patterns: (a) MCM41, (b) APS-MCM41, (c) Thio-APS-MCM41, (d) $\mathrm{CuO}_{2}$ (acac)-Thio-APS-MCM41

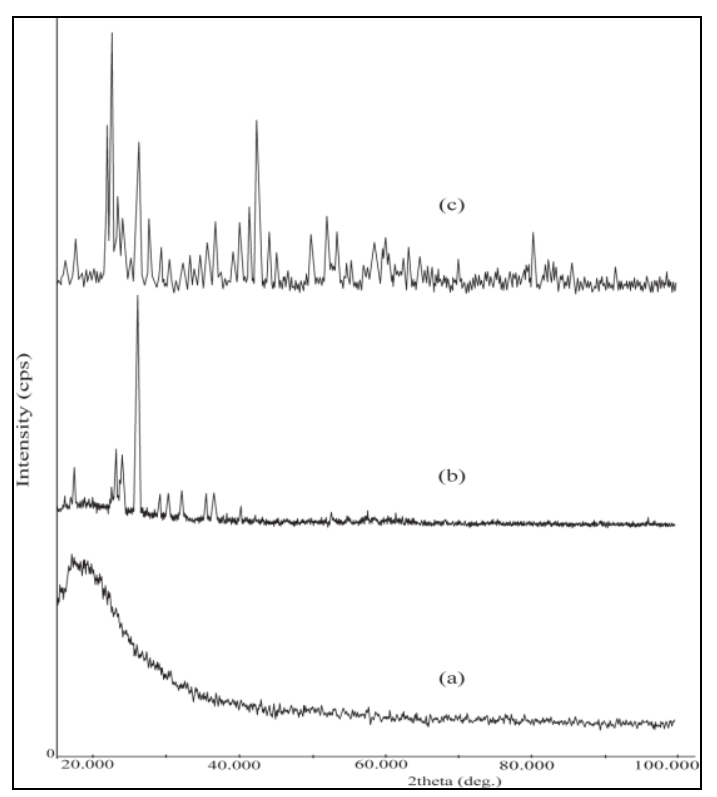

Fig. 3. High angle XRD patterns: (a) APS-MCM41, (b) Thio-APS-MCM41, (c) $\mathrm{CuO}_{2}$ (acac)-Thio-APS-MCM41

\section{B. Single Point BET}

Table I displays the results of the synthesized material from the single-point BET analysis. Results show clearly that the BET surface area of MCM41 is $982.56 \mathrm{~m}^{2} / \mathrm{g}$. It has been reported that mesoporous silica materials have surface area is above $600 \mathrm{~m}^{2} / \mathrm{g}$ [11]. From the data, it can be seen that the BET surface area of APS-MCM41, Thio-APS-MCM41 and $\mathrm{CuO}_{2}$ (acac)-Thio-APS-MCM41 reduces remarkably compared to MCM41, corresponding to the various groups that were successfully grafted. The decrease in surface area suggests that mesoporous framework has turned amorphous. Once a mesoporous framework collapsed from its crystalline structure to amorphous phase, the surface area is decreased significantly. Meanwhile, the pore volume of synthesized materials is significantly decreased due to the existence of high amorphous phase in Thio-APS-MCM41and $\mathrm{CuO}_{2}$ (acac)-Thio-APS-MCM41 sample blocked the pore structure of sample to give lower pore volume.

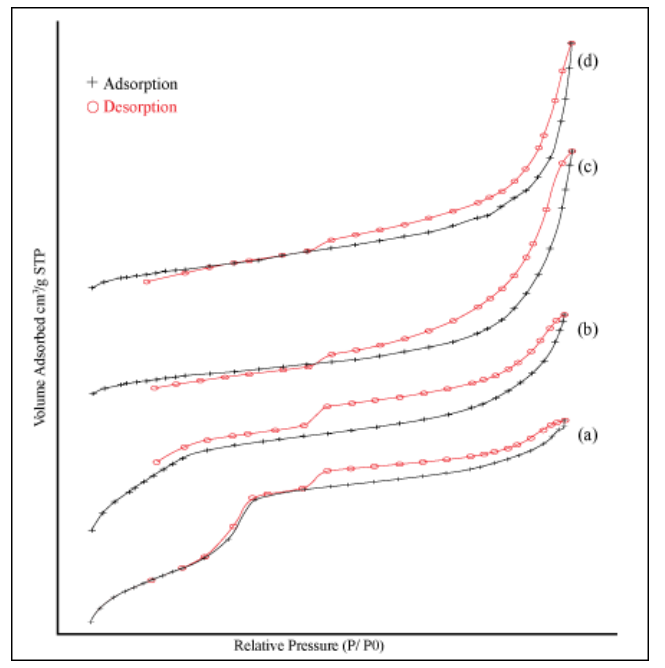

Fig. 4. Nitrogen adsorption isotherms: (a) MCM41, (b) APS-MCM41, (c) Thio-APS-MCM41, (d) $\mathrm{CuO}_{2}$ (acac)-Thio-APS-MCM41

TABLE I: SURFACE PROPERTIES OF SYNTHESIZED MATERIALS

\begin{tabular}{|l|c|c|c|}
\hline Samples & $\begin{array}{c}\text { BET } \\
\text { surface } \\
\text { area }\left(\mathrm{m}^{2} / \mathrm{g}\right)\end{array}$ & $\begin{array}{c}\text { Average } \\
\text { pore } \\
\text { diameter } \\
(\AA)\end{array}$ & $\begin{array}{c}\text { Average } \\
\text { pore } \\
\text { volume } \\
\left(\mathrm{cm}^{3} / \mathrm{g}\right)\end{array}$ \\
\hline MCM41 & 982.56 & 35.99 & 0.88 \\
\hline APS-MCM41 & 286.91 & 30.79 & 0.22 \\
\hline Thio-APS-MCM41 & 14.44 & 99.35 & 0.04 \\
\hline $\begin{array}{l}\mathrm{CuO}_{2} \text { (acac)-Thio-APS-MCM4 } \\
1\end{array}$ & 10.01 & 95.26 & 0.02 \\
\hline
\end{tabular}

\section{Nitrogen Adsorption}

Nitrogen adsorption isotherms of synthesized materials are presented in Fig. 4. The isotherms were of type IV which shows a typical mesoporous solid, according to IUPAC classification [12]. All the samples exhibited strong adsorption at a relative pressure in the range of $0.2 \leq \mathrm{P} / \mathrm{P}_{0} \leq$ 0.5 . Adsorption at low pressure $\left(\mathrm{P} / \mathrm{P}_{0}<0.25\right)$ is accounted for by monolayer-multilayer adsorption of $\mathrm{N}_{2}$ on the wall of mesopores. At $\mathrm{P} / \mathrm{P}_{0}=0.25-0.45$, the figure show a sharp step capillary condensation, suggesting a narrow pore distribution. Meanwhile, at $\mathrm{P} / \mathrm{P}_{0}=0.5$, the accessible pores are totally filled with adsorbate and the isotherm reaches a plateau that remains fairly invariant as $\mathrm{P} / \mathrm{P}_{0}$ approaches unity. Besides, the isotherms also show the characteristic of capillary condensation into uniform mesopores and possessed good mesoporous structural ordering. No change was observed in isotherm (c) and (d) after immobilization of copper indicating that the mesoporous structure was retained. However, 
nitrogen uptake decreases due to the presence of bulky materials inside the pores [13]. The narrow pore size distribution as seen from Fig. 5 revealed a uniform mesoporosity. The pore distribution become broader indicated the ordering of samples decreased as the content of heteroatom increased.

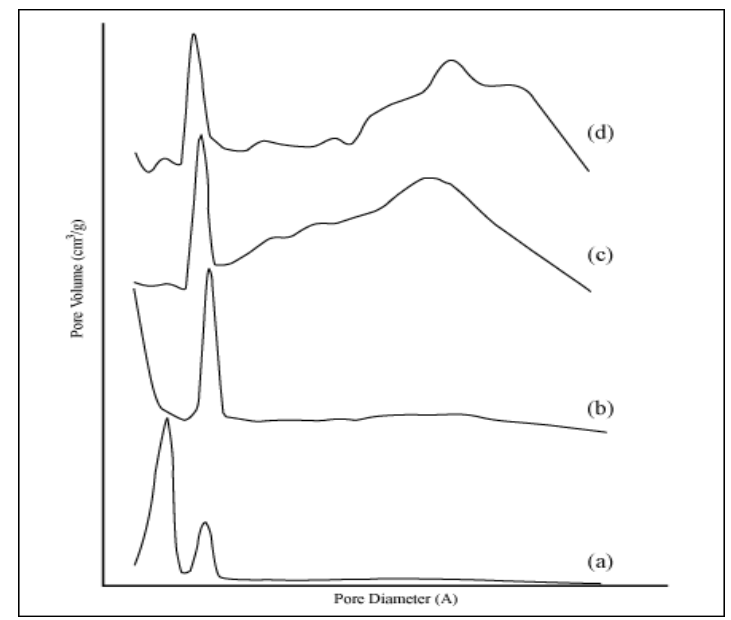

Fig. 5. Pore size distribution: (a) MCM41, (b) APS-MCM41, (c) Thio-APS-MCM41, (d) $\mathrm{CuO}_{2}$ (acac)-Thio-APS-MCM41

\section{FT-IR Spectra}

FT-IR spectra of synthesized materials are shown in Fig. 6 and 7. The samples were recorded at ambient condition with $\mathrm{KBr}$ technique in the region of $4000-400 \mathrm{~cm}^{-1}$. The FT-IR spectrum of the uncalcined MCM41 (Fig. 6a) shows the peak of surfactant around $2921 \mathrm{~cm}^{-1}$ and $2852 \mathrm{~cm}^{-1}$ corresponding to $\mathrm{n}-\mathrm{CH}$ and $\mathrm{d}-\mathrm{CH}$ vibrations of the surfactant molecules. But these peaks are absent in the sample heated at $550{ }^{\circ} \mathrm{C}$ which is celled calcinations process due to by the removal of template (Fig. 6b). It means the surfactant was successfully removed from the framework of MCM41. The major peaks of MCM41 were observed in the FT-IR spectra of calcined MCM41 that consist of asymmetrical Si-O-Si stretching vibration in the range between $1200-1000 \mathrm{~cm}^{-1}$ which is $1100 \mathrm{~cm}^{-1}$, symmetrical Si-O-Si stretching vibration at $802 \mathrm{~cm}^{-1}, \mathrm{Si}-\mathrm{O}-\mathrm{Si}$ bending at $460 \mathrm{~cm}^{-1}$ and $\mathrm{Si}-\mathrm{OH}$ stretching vibration at 970 $\mathrm{cm}^{-1}[14,15]$. These peaks indicated that MCM41 was successfully synthesized. Fig. $6 \mathrm{c}$ shows the FT-IR spectra of MCM41 after being modified with APTES. The FT-IR spectra shows the presence of $\mathrm{N}-\mathrm{H}$ bending vibration at 790 $\mathrm{cm}^{-1},-\mathrm{NH}_{2}$ symmetric bending at $1553 \mathrm{~cm}^{-1}$ and $\mathrm{C}-\mathrm{N}$ bond at $1639 \mathrm{~cm}^{-1}$ that are absent in neat MCM41. Moreover, the asymmetric vibration of the $\mathrm{CH}_{2}$ groups of the propyl chain of the sylilating agent at $2944 \mathrm{~cm}^{-1}$, indicates the successful grafting of organic amine onto the pore of MCM41. Fig. 7 shows the spectra for thiouracil (a), Thio-APS-MCM41 (b) and $\mathrm{CuO}_{2}$ (acac)-Thio-APS-MCM41 (c). The spectra of 2-thiouracil are known to contain two very specific bands of IR absorption [16] at $1710 \mathrm{~cm}^{-1}$ that corresponds to $\mathrm{C}=\mathrm{O}$ stretching mode and $\mathrm{C}=\mathrm{S}$ to $\mathrm{N}$ vibration at $1220 \mathrm{~cm}^{-1}$. Both of these two bands are present in IR spectrum after encapsulated APS-MCM41 with thiouracil and metal. Besides, the Si-O-Si bond also remained even after modification with thioracil and metal. The bands at $628 \mathrm{~cm}^{-1}$ and $691 \mathrm{~cm}^{-1}$ represents the $\mathrm{Cu}=$ $\mathrm{O}$ vibrations [15]. This results indicates that, the Thio-APS-MCM41 and $\mathrm{CuO}_{2}$ (acac)-Thio-APS-MCM41 is successfully synthesized.

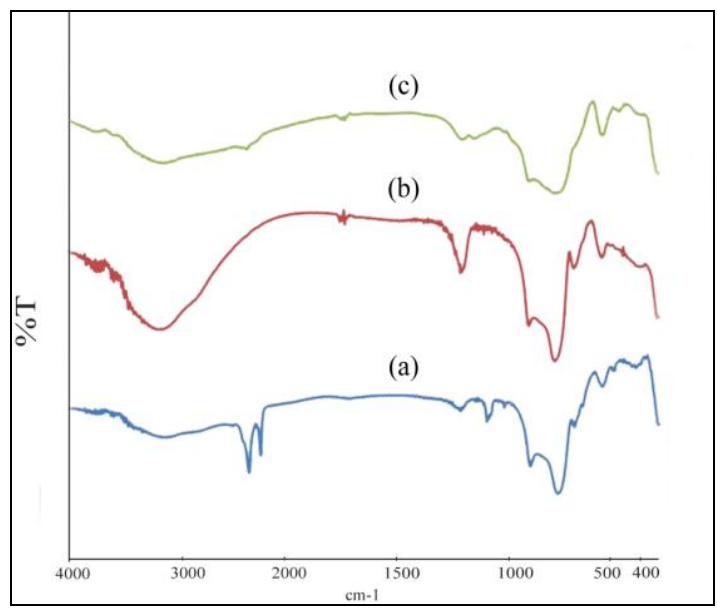

Fig. 6. FT-IR spectra: (a) uncalcined MCM41, (b) MCM41, (c) APS-MCM41

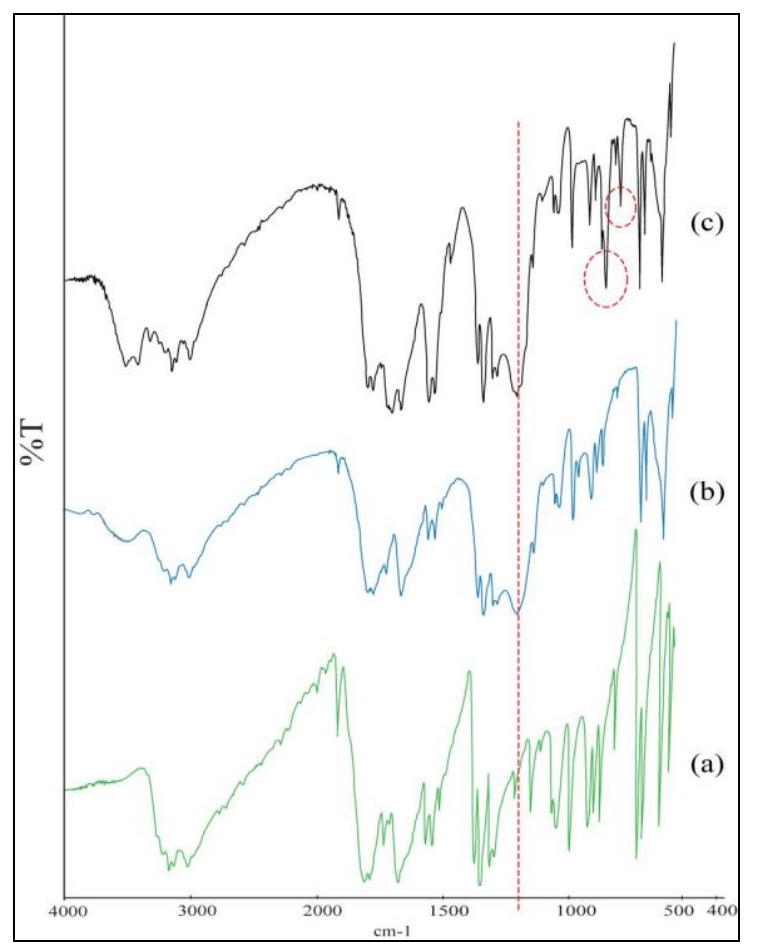

Fig. 7. FT-IR spectra: (a) Thiouracil, (b) Thio-APS-MCM41, (c) $\mathrm{CuO}_{2}$ (acac)-Thio-APS-MCM41

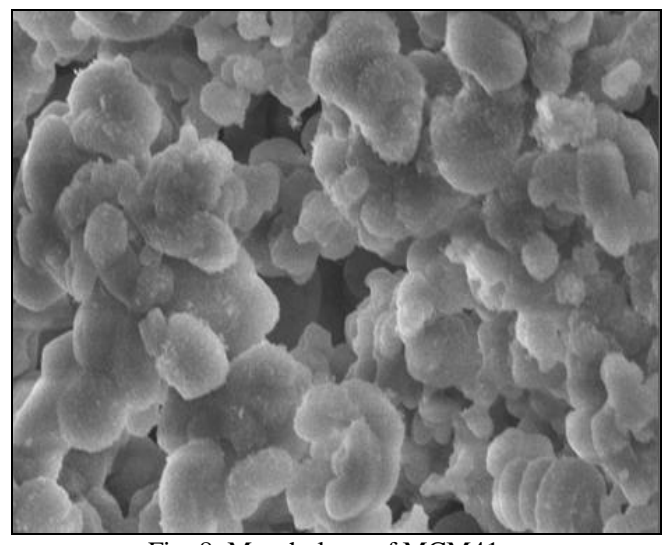

Fig. 8. Morphology of MCM41

\section{E. Scanning Electron Microscopy (SEM)}

Fig. 8 and 9 display the FESEM micrograph of pure 
MCM41 and $\mathrm{CuO}_{2}$ (acac)-Thio-APS-MCM41, respectively. The FESEM micrograph reveals that the particle morphology of both samples consists of agglomerated particles of 1 to 3 $\mu \mathrm{m}$ in diameter.

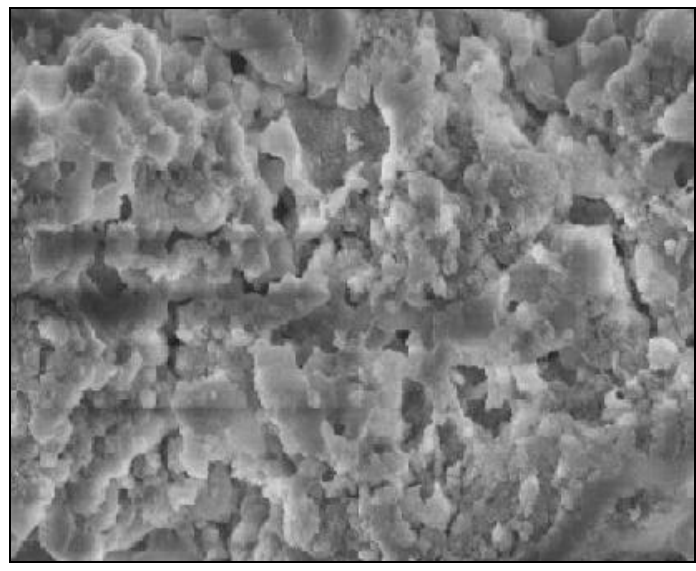

Fig. 9. Morphology of $\mathrm{CuO}_{2}$ (acac)-Thio-APS-MCM41

\section{F. Elemental Analyzer}

According to elemental analysis results, the carbon contents for the Thio-APS-MCM41 and $\mathrm{CuO}_{2}$ (acac) -Thio-APS-MCM41 are determined to be 23.9 and 24.4 wt. $\%$, respectively. Considering the theoretic carbon contents of Thio-APS-MCM41 and $\mathrm{CuO}_{2}$ (acac)-Thio-APS-MCM41 are 25.6 and $23.9 \mathrm{wt} \%$, this indicates that the composition of prepared samples coincide well with their molecular formula.

\section{CONCLUSION}

A $\mathrm{CuO}_{2}(\mathrm{acac})_{2}$ supported on functionalized-MCM41 containing thiourea ligand was successfully synthesized. Characterization results indicated that the thiourea ligand and copper acetate was encapsulated into the pores of functionalized-MCM41. The structure properties of synthesized materials all suggested potential application in catalytic field.

\section{ACKNOWLEDGMENT}

This work has been supported by the Ministry of Science, Technology and Innovation Malaysia (MOSTI) for financial support through funding 600-RMI/ST/FRGS5/3/Fst (41/2010) and very thanks to the Faculty of Pharmacy, Universiti Teknologi MARA and Faculty of Science, Universiti Teknologi Malaysia for cooperation in completing this work.

\section{REFERENCES}

[1] C. T. Kresge, M. E. Leonowicz, W. J. Roth, J. C. Vartuli, and J. S. Beck, "Ordered mesoporous molecular sieves synthesized by liquid crystal templating mechanism,” Journal Nature, vol. 359, pp. 710-712, 1992.

[2] G. J. de A.A. Soler-Illia, C. Sanchez, B. Lebeau, and J. Patarin, "Chemical strategies to design textured materials: from microporous and mesoporous oxides, to nanonetworks and hierarchical structures,' Chem. Rev., American Chemical Society, 102, pp. 4093-4138, 2002.

[3] Y. Kong, X. Guo, F. Zhang, S. Jiang, J. Wang, Y. Lu, and Q. Yan, "Synthesis of Ti-containing MCM41 using Ti( $\left.\mathrm{SO}_{4}\right)_{2}$ as Ti source," Material Letters, 59, pp. 3099-3103, 2005.

[4] R.S. Robert, A. Rafael, A.D. James, and W.R. Thatcher, "Vapor-phase silylation of MCM-41 and Ti-MCM41," Micropor. Mesoporm Mater., 66, pp. 53-67, 2003

[5] X. S. Zhaoand G. Q. Lu, "Modification of MCM41 by surface silylation with trimethylchlorosilane and adsorption study," J. Phys. Chem, B., vol. 102, pp. 1556-1561, 1998.
[6] A. Taguchi and F. Schüth, "Ordered mesoporous materials in catalysis," Micropor. Mesopor. Mater, vol. 77, pp. 1, 2005.

[7] T. Shahram, M. Majid, M. Valiollah, M. Iraj, and G. Kamal, "Alkene epoxidation catalyzed by molybdenum supported on functionalized MCM41 containg N-S chelating Schiff base ligand," Catal. Comm., vol. 10, pp. 853-858, 2009.

[8] Y. Yuan, W. Cao, and W. Weng, " $\mathrm{CuCl}_{2}$ immobilized on amino-functionalized MCM41 and MCM48 and their catalytic performance toward the vapor phase oxycarbonylation of methanol to dimethylcarbonate," Journal of Catalysis, vol. 228, pp. 311-320, 2004.

[9] S. Biz and M. L. Occelli, "Synthesis and characterization of mesostructured materials," Catal. Rev. Sci. Eng., vol. 40, pp. $329,1998$.

[10] K. M. Parida, D. Rath, and S. S. Dash, "Synthesis, characterization and catalytic activity of copper incorporated and immobilized mesoporous MCM-41 in the single step amination of benzene," $J$. Molec. Catal. A: Chem., vol. 318, pp. 85-93, 2010.

[11] S. Endud and K. L. Wong, "Mesoporous silica MCM48 molecular sieve modified with $\mathrm{SnCl}_{2}$ in alkaline medium for selective oxidation of alcohol," Micropor. Mesopor. Mater., vol. 101, pp. 256-263, 2007.

[12] S. Brunauer, L.S. Deming, W. S. Deming, and E. Teller, "On a theory of the van der waals adsorption of gases," J. Am. Chem. Soc., vol. 62, no. 7, pp. 1723-1732, 1940.

[13] H. Yang, G. Zhang, X. Hong, and Y. Zhu, "Dicyano-functionalized MCM-41 anchored-palladium complexes as recoverable catalysts for Heck reaction," J. Mol. Cat. A., vol. 210, pp. 143-8, 2004.

[14] E. M. Flanigen, H. Khatami, and H. A. Szymanski, "Infrared structural studies of zeolite frameworks," In: E.M. Flanigen, L.B. Sand (Eds.). Molecular Sieve Zeolites, ACS Adv. Chem. Ser, 1971, vo. 101, pp. 201-227.

[15] R. Takahashi, S. Sato, T. Sodesawa, M. Kawakita, and K. Ogura, "High surface-area silica with controlled pore size prepared from nanocomposite of silica and citric acid," J. Phys. Chem. B., vol. 104, pp. $12184,2000$.

[16] L. Chmielarz, P. Kustrowski, R. Dziembaj, P. Cool, and E. F. Vansant, "Catalytic performance of various mesoporous silicas modified with copper or iron oxides introduced by different ways in the selective reduction of NO by ammonia," Appl. Catal. B: Env., vol. 62, pp. 369-380, 2006.

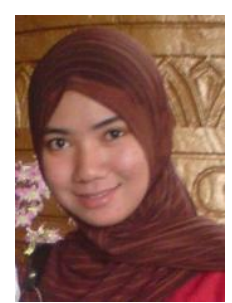

Amirah Ahmad was born in Terengganu, Malaysia, in June 16, 1988. She received B.Sc (2010) in Applied Chemistry from Universiti Teknologi MARA, Shah Alam, Malaysia. She is post graduate student (M. Sc) at Universiti Teknologi MARA in inorganic chemistry. Her research is focused on synthesis of new catalyst from mesoporous MCM41 that containing thiourea complex, used in oxidation reaction.

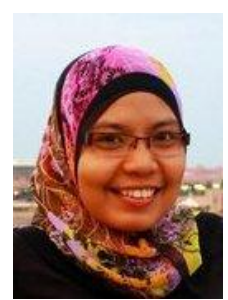

Hamizah Md. Rasid was born in Johor, Malaysia, on July 10, 1986. She received B. Sc (2008) and M.Sc (2010) in Chemistry from Universiti Teknologi Malaysia, Johor, Malaysia. She is specialized in heterogeneous catalysis. Currently, she is a lecturer at Universiti Teknologi MARA.

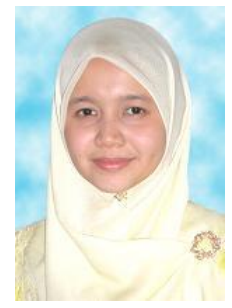

Karimah Kassim was born in Selangor, Malaysia, in January 24, 1978. She received Ph.D (2010) in Coordination Chemistry from Universiti Teknologi MARA, Shah Alam, Malaysia. Her research is focused on synthesis and characterization of macrocyclic and Schiff Base complexes. She has studied the magnetic properties, biological activities, corrosion inhibition studied and conductivity studies for the synthesized compound. 